\title{
The Impact of Search Engine Optimization on The Visibility of Research Paper and Citations
}

\author{
Asim Shahzad ${ }^{\#}$, Nazri Mohd Nawi ${ }^{\#}$, Norhamreeza Abd Hamid", Sundas Naqeeb Khan\#, \\ Muhammad Aamir ${ }^{\#}$, Arif Ullah ${ }^{\#}$, Salfarina Abdullah* \\ \# Faculty of Computer Science and Information Technology, Universiti Tun Hussein Onn Malaysia, Malaysia \\ * Faculty of Computer science and Information Technology, Universiti Putra Malaysia, Malaysia \\ E-mail:nazri@uthm.edu.my
}

\begin{abstract}
The initial criteria for evaluating a researcher's output is the number of papers published. Furthermore, for the measurement of author's research quality, the number of citations is significant. Typically, citations are directly linked with the visibility of a research paper. Many researches had shown that the visibility of a research paper can be improved further by using the search engine optimization techniques. In addition, some research already proved that the visibility of an article could improve the citation results. In this article, we analysed the impact of search engine optimization techniques that can improve the visibility of a research paper. Furthermore, this paper also proposing some strategies that can help and making the research publication visible to a large number of users.
\end{abstract}

Keywords - H-Index, research impact, search engine optimization, citations, research marketing, research visibility.

\section{INTRODUCTION}

Citation reveals the number of times other articles have used and referred to an article. The citations measure the significance of the information contained in a research paper [1]. "The more frequently a paper becomes cited, the bigger its effect on the field" is an underlying supposition of citation analysis [2] [3]. The primary indicator of the importance of a research output is direct citation [4] [5].

Besides, in different universities ranking criteria, the number of citations has more than twenty percent share [6] [7]. Therefore, the university's researchers are encouraged by the universities to write high standards research articles which can get significant citations and would reach the largest possible audience [3]. The researchers are agreed that by increasing the visibility of a research article using any method will results in high access and huge citation impact [5] [6] [8] [9] [10].

A research article has the high possibility of becoming cited most whenever got increased visibility [11]. As all the research articles which have the open-access to the users got the more visibility that's why these articles are getting more citations. In a research study, Antelman proved that openly available research papers have the biggest research impact as compared to the research papers which do not have the open and free access to the users [6]. Now, most of the authors realized the importance of visibility and importance of citation, so they are highly motivated to publish their research in free and open access repositories [12].

The biggest advantage of improving the visibility and publishing in open access journals is that it will boost up to ten percent in citations [13]. Another researcher investigated the significance of visibility and importance of open and free online availability of research papers [14]. Nevertheless, with the visibility of articles, the quality of articles is also important for the greater number of citations [15].

High visibility of an article can be achieved by using search engine optimization (SEO) techniques and existing methods which can improved visibility as well as will increase the number of citations [16]. Researchers are spending a lot of time and efforts in writing their research papers for publication. But still, publishing an article in leading journal with high impact factor cannot guarantee the high impact on the citation. There is a robust relationship between the number of times a research paper is cited and the possibility that the research paper is online [14].

Self-archiving of an article by the author can highly increase the visibility of the paper. Storing the articles and research results in researcher's website, blog, web pages or institutional repositories is known as self-archiving [9]. The main advantage of Self-archiving benefits is that the article is available for free and accessible by the larger number of 
audience. By applying the SEO (Search Engine Optimization) techniques to these self-archiving repositories it will make the articles more visible to all the audiences by simple Yahoo, Bing, and Google search [17].

Another significant benefit of self-archiving the articles and applying the SEO techniques on these freely available materials is that it will easily be found on researcher's website by simple Google search rather than in any other repository or free open access journal [6]. Besides this, institutional and organizational repositories can make the research papers more visible and increase the possibilities of citation by other researchers and scholars [18].

Therefore, for the best visibility and getting more citation SEO techniques should be applied because more visibility means: Article is cited sooner, a huge number of citations and citations continue at a notable ratio for a prolonged period [19]. Due to less visibility, some very senior researchers from African universities do not have the significant citation impact [20]. By insertion of Keywords in a research article, writing the search engine friendly abstract and title can maximize visibility after it is published [21] [22].

Furthermore, by making some adjustments in where to submit, how to present and publish the research results, researchers can become productive, highly known for their work, and strongly connected to the researchers of own professional community [23]. There are a lot of SEO techniques for enhancing the readership and visibility of research articles. Efficient use of these SEO techniques can result in increased visibility which will directly affect the article citations [3].

In this study, we explored some of the SEO techniques for increasing the visibility of research articles. This paper also demonstrates that the use of SEO techniques can directly affect the visibility of a research article as will be discussed further in the next section.

\section{MATERIAL AND METHOD}

Normally, for any research paper that appears on top in search engine result pages, then it will receive researcher's attention and will get the download counts with citations. Search engine optimization techniques as listed in Table 1 can be used for increasing the visibility of research papers to make the articles available to a broader audience [24].

Search Engine Optimization (SEO), is the method of increasing the visibility of website, web page, web profile or research article on original (natural, organic or unpaid) search engine result pages (SERPs), by including search engine (SE) friendly components. An effective SEO will have, precisely selected, relevant keywords by which the on-article optimization will be designed to make prominent for SE algorithms [25].

SEO for a research article is consists of two major areas: on-article, and off-article optimization [26]. On-article optimization refers to research article elements which comprise a research article such as, research paper's title, abstract, sub-titles in a research paper and text. Off-page optimization mainly refers to backlinks (incoming links to the research articles, and researcher profile, which is being optimized, from other relevant websites) [25].
Table 1: Search engine optimization and other techniques used to make the research articles visible to a broader audience.

Search Engine Optimization Techniques for Visibility On Article

Optimization

\begin{tabular}{ll}
\hline & Research Paper's Title Optimization \\
\cline { 2 - 2 } $\begin{array}{l}\text { Off Article } \\
\text { Optimization }\end{array}$ & Keywords consistency and density \\
\hline & $\begin{array}{l}\text { Researcher's website and } \\
\text { consistency in the author's name. } \\
\text { Get Social on Social Networks }\end{array}$ \\
& $\begin{array}{l}\text { Researcher's contributions on } \\
\text { Wikipedia }\end{array}$ \\
\hline & $\begin{array}{l}\text { Backlink creation for research } \\
\text { articles }\end{array}$ \\
\hline Other Techniques for Improving the Visibility \\
\hline & $\begin{array}{l}\text { Publish research papers in an open- } \\
\text { access journal }\end{array}$ \\
\hline $\begin{array}{l}\text { Sharing research outcomes except } \\
\text { manuscript: }\end{array}$ \\
\hline $\begin{array}{l}\text { Few more tools for improving } \\
\text { visibility }\end{array}$ \\
\hline
\end{tabular}

(a) Research paper's title and keywords: Select research paper's title and keywords very intelligently. Relevant keywords for the Research topic and search engine friendly title is playing a significant role in the visibility of an article in SERP,s [27]. The title and keywords can profoundly influence the possibilities of getting picked up when searched, read, and cited. Search engines, Journals, and abstracting and indexing services, classify the research articles by keywords. The relevant list of keywords will, therefore, assure accurate indexing and help showcase the research work to attract the groups who are already working the relevant field. The best practice for selecting keywords is, select those words which are repeatedly used in the research paper but preferably not repeated in abstract or title [22]. The title of the research paper should be clear, catchy and straightforward while describing the research work appropriately. It is essential to think about keywords that readers can use to search for research articles include such kind of words in the article, and the best practice is to avoid the jargon and abbreviations.

(b) Researcher's website and consistency in the author's name: Author needs to develop the personal website/blog where he/she can share his/her research contributions, thoughts, ideas, key findings, promote his/her research work, or write about how his/her work is necessary for others and influenced others. On personal website/blog, he/she can list all of his research papers and provide the abstract to papers and download links [24]. This will improve the visibility of his/her research articles and will improve the citations. The other important thing for any research is unique author identifier. Every researcher should get a unique author identifier (Open Researcher and Contributor ID, ORCID) to differentiate himself and his work from other researchers. The output of a researcher can easily be retrieved if he/she is using the uniform name throughout a research career [27]. General issues with consistent author name include legal name 
changes, inconsistent name formats, famous names or incredibly similar names, which can make it tough to correlate research output to the right author [28].

(c) Get Social on Social Networks: It is proved from the research studies that there is a statistically dominant relationship among social media mentions such as tweets, posts, blogs, and citation counts. The researcher should be engaged with groups and individuals beyond academia, including decision makers and system influencers, and with individuals with common interests via Twitter, Facebook, Academia.edu, LinkedIn, and other groups, sharing research work and discussing on articles. Proactive engagement is required with researchers, individuals, and groups who might be interested in the similar research work. The author can engage everyone who is interested in his/her research work through different social media networks to keep them informed about new research findings [29].

A Twitter account can help the author to promote his/her research contributions. Authors can tweet about different things: research contribution, publications, and findings, views on latest research trends, and news relevant to research interest. Same can be done with the Facebook posts. The hashtag (\#) is used for classification of contents on social media. A phrase or word preceded by a hashtag (\#), used within a message to identify a topic of interest or keyword and facilitate a search for it. Adding a hashtag to post means social network will index it and it will become discoverable/searchable by other users [30]. It will allow one to engage and connect with other social media users based on a common interest. The exact and more focused hashtag can profoundly engage the targeted audience.

Another multidisciplinary and essential academic networking site is Academia.edu. It is best for showcasing publications, achievements, and expertise due to its strong profile and perfect resume feature. Available measures of impact include counts for document views, profile views, unique visitors, document downloads, the geographic division of users and referrals, backlinks to documents. The last but not least is LinkedIn which is one of social networking website for professionals in which professionals can create their profiles, connect with network and peers. The researcher can list all their research publications and other research outcomes, skills, experiences, past and current positions.

\section{(d) Researcher's contributions on Wikipedia:}

Thousands of editors are contributing to the Wikipedia pages, and every contributor is coming up with something new whether it is technical expertise or researching skills, but more importantly an enthusiasm to benefit others [31]. Nobody owns anything present on Wikipedia, therefore if a person sees any issue or want to add some more details on the relevant topic, he/she is allowed to do so.

This is an excellent opportunity for the author to introduce him/her self; the author can write a new research article from scratch or edit the existing research articles relevant to his/her area of interest and can add the reference of his/her research articles in the references section of Wikipedia article. This will increase the visibility of his research work to the other individuals and similar research groups. (e) Backlink creation for research articles: When a research article links to any website, it is called a backlink for the research article [29]. SEO experts believe that backlinks are essential for the visibility of contents and significant metric for placing the research articles on the top in SERPs. A research article with many backlinks tended to rank top on all the big search engines, including Bing and Google. Many methods can be used for research article's backlink creation. Social media can be used for backlink creation [28]. For this purpose, the author needs to identify the relevant discussion groups on social media [29]. After finding the list of relevant research discussion groups, the author can take part in discussions and can tell research community about his research contributions in the area. Similarly, the author can search for relevant online discussion forums and participate in discussions and can add the link to his/her research articles for references. The author can also write the guest posts for the top-ranked research blogs and can add the links to his/her research articles. By creating the quality backlinks in relevant forums, blogs and discussion groups' author can increase the visibility of research articles and his research profile.

\section{RESUTS AND DISCUSSION}

Apart from SEO techniques, there are also available some other techniques that can be use in which can increase the visibility of research articles for getting more citations.

(a) Publish research papers in an open-access journal: For more excellent visibility author can make articles open access. There is an advantage of open access articles that articles are accessible to every reader for free of cost including policymakers, and this improves the possibility of being cited by the scholars [32]. Authors can also self-archive the papers on their websites for direct and quick open access to it. Academia.edu and ResearchGate are types of free online repositories. On these repositories, the author can share their published work for broader circulation and greater visibility. Authors can also share their research work on institutional repositories, but before sharing the published work on open access repository, it is essential to check with the publisher for their self-archiving policy.

(b) Sharing research outcomes except manuscript: Besides published research papers every researcher has the evidence of his/her research activity which includes working papers, preprints, presentations, conference papers, reports, print copy, protocols, videos and research data [29]. The author can put all this data on public repositories for increasing the visibility of research work. The research community is very frequently using the SlideShare and Scribd so the author can upload all the slides for improved visibility. F1000 Research can be used for depositing the research posters and presentations. Research data can be published on figshare, Zenodo or Data Dryad which it will improve the visibility [33].

(c) Few more tools for improving visibility: presenting the research results at a conference or in educational meetings can highlight the author, which can improve the visibility of author's papers. Researchers can promote their research findings together with a measure of their impact with tools 
like ImpactStory and Kudos which are powerful tools for researchers. These tools enable the researchers to showcase their research articles by creating links to full texts and any other supplementary information like a layman language explanation, short title, links to relevant contents, for example, videos, code, slides or any other data [33]. By using these tools, the author can share contents on social media and can also monitor and track the results of every activity through the posts, number of tweets, downloads, visits, and views. Google Scholar is another potent tool for researcher which can improve the visibility of researcher and research contributions. Anyone can create the Google Scholar profile for free and can take the advantages of Google Scholar [34].

\section{CONCLUSION}

From this research study, we can conclude that it is possible that a research article may not be written extraordinarily and may not be published in a leading journal, but by applying the appropriate SEO and other techniques for improving visibility, the paper still can get sufficient citations and worldwide acknowledgment. The author should learn the techniques of using SEO, Social media and other tools to enhance the visibility of their research work. Once author successfully increased the visibility of research article using proposed methods the citations will rise at a notable rate. Therefore, a little hard work and some simple SEO techniques can improve the article's visibility.

\section{ACKNOWLEDGMENT}

The authors would like to thank Universiti Tun Hussein Onn Malaysia (UTHM) Ministry of Higher Education (MOHE) Malaysia for financially supporting this Research under Trans-displinary Research Grant Scheme (TRGS) vote no. T003. This research also supported by GATES IT Solution Sdn. Bhd under its publication scheme.

\section{REFERENCES}

[1] Fooladi, Masood, et al. "Does criticisms overcome the praises of journal impact factor?." arXiv preprint arXiv:1305.0379 (2013).

[2] Martínez, M. A., et al. "H-Classics: characterizing the concept of citation classics through H-index." Scientometrics 98.3 (2014): 1971-1983.

[3] Garfield, Eugene, and Robert King Merton. Citation indexing: Its theory and application in science, technology, and humanities. Vol. 8. New York: Wiley, 1979.

[4] Shotton, David. "Open citations." Nature 502.7471 (2013): 295-297.

[5] Priem, Jason. "Scholarship: Beyond the paper." Nature 495.7442 (2013): 437-440.

[6] Usher, Alex, and Massimo Savino. "A global survey of university ranking and league tables." Higher Education in Europe 32.1 (2007): $5-15$.

[7] Taylor, Paul, and Richard Braddock. "International university ranking systems and the idea of university excellence." Journal of Higher Education Policy and Management 29.3 (2007): 245-260.

[8] Hardy, Rachel, et al. "Open access citation information." (2005).

[9] Ertürk, Korhan Levent, and Gökhan Şengül. "Self Archiving in Atılım University." E-Science and Information Management (2012): 79-86.

[10] Dalton, Michelle. "A dissemination divide? The factors that influence the journal selection decision of Library \& Information Studies (LIS) researchers and practitioners." Library and Information Research 37.115 (2013): 33-57.
[11] Egghe, Leo, Raf Guns, and Ronald Rousseau. "Measuring co-authors' contribution to an article's visibility." Scientometrics 95.1 (2013): 55-67.

[12] Jayaprakash, K., A. Rekha, and S. Rajendiran. "Open Access Journals-A Study." IOSR Journal Of Humanities And Social Science 8 (2013): 07-09.

[13] McCabe, Mark J., and Christopher M. Snyder. "Does online availability increase citations? Theory and evidence from a panel of economics and business journals." Review of Economics and Statistics 97.1 (2015): 144-165.

[14] Lawrence, Steve. "Free online availability substantially increases a paper's impact." Nature 411.6837 (2001): 521.

[15] Kurtz, Michael J., et al. "The effect of use and access on citations." Information Processing \& Management 41.6 (2005): 1395-1402.

[16] Ivanović, Mirjana, et al. "Computer Science and Information Systems: Publishing an international Open Access journal in a developing country." (2013).

[17] Koulouris, Alexandros, et al. "Institutional repository policies: best practices for encouraging self-archiving." Procedia-Social and Behavioral Sciences 73 (2013): 769-776.

[18] Miguel, Sandra, Zaida Chinchilla-Rodriguez, and Félix de Moya Anegón. "Open access and Scopus: A new approach to scientific visibility from the standpoint of access." Journal of the Association for Information Science and Technology 62.6 (2011): 1130-1145.

[19] Patel, Vanash M., et al. "Enhancing the h index for the objective assessment of healthcare researcher performance and impact." Journal of the Royal Society of Medicine 106.1 (2013): 19-29.

[20] Rotich, Daniel C., and Joseph J. Musakali. "Publish or perish remaining academically relevant and visible in the global academic scene through scholarly publishing." Conference and Programme Chairs. 2013

[21] Norman, Emma R. "Maximizing journal article citation online: readers, robots, and research visibility." Politics \& Policy 40.1 (2012): 1-12.

[22] Tate, Joanna. An evaluation of SOAS Research Online, the Institutional Repository of the School of Oriental and African Studies. Diss. City University, 2010.

[23] Pfirman, S., et al. "Maximizing Productivity and Recognition, Part 1: Publication, Citation, and Impact." (2007).

[24] Tripathy, J. P. et al. "Ten Tips to Improve the Visibility and Dissemination of Research for Policy Makers and Practitioners." Public Health Action 7.1 (2017): 10-14. PMC. Web. 10 Oct. 2017.

[25] Zhang, Sonya, and Neal Cabage. "Search Engine Optimization: Comparison of Link Building and Social Sharing." Journal of Computer Information Systems 57.2 (2017): 148-159.

[26] Baye, Michael R., Babur De los Santos, and Matthijs R. Wildenbeest. "Search engine optimization: what drives organic traffic to retail sites?." Journal of Economics \& Management Strategy 25.1 (2016): 6-31.

[27] Ale Ebrahim, Nader, et al. "Effective strategies for increasing citation frequency." (2013).

[28] Zhao, Chunshui, Jun Yan, and Ning Liu. "Improve Web Search Ranking by Co-Ranking SVM." Natural Computation, 2008. ICNC'08. Fourth International Conference on. Vol. 2. IEEE, 2008.

[29] Zhang, Sonya, and Neal Cabage. "Search Engine Optimization: Comparison of Link Building and Social Sharing." Journal of Computer Information Systems 57.2 (2017): 148-159.

[30] Bong, Yiibonn, and Nader Ale Ebrahim. "Increasing Visibility and Enhancing Impact of Research." (2017).

[31] Milne, David, and Ian H. Witten. "An open-source toolkit for mining Wikipedia." Artificial Intelligence 194 (2013): 222-239.

[32] Salager-Meyer, Françoise. "Writing and publishing in peripheral scholarly journals: How to enhance the global influence of multilingual scholars?." Journal of English for Academic Purposes 13 (2014): 78-82.

[33] Leonardi, Paul M. "Social media, knowledge sharing, and innovation: Toward a theory of communication visibility." Information systems research 25.4 (2014): 796-816.

[34] Lateef, A., A. T. J. Ogunkunle, and G. O. Adigun. "Google scholar citation in retrospect: Visibility and contributions of African scholars." COLLNET Journal of Scientometrics and Information Management 10.2 (2016): 219-236. 